\title{
Prática na comunidade mediada pelo Arco de Maguerez: problematização em uma residência multiprofissional em saúde mental
}

\author{
Maguerez Arch-Mediated Community Practice: Problematization in a multiprofessional \\ residency in mental health
}

\author{
Práctica comunitaria mediada por el arco de Maguerez: problematización en una \\ residencia multiprofesional en salud mental
}

José de Siqueira Amorim Júnior ${ }^{1 *}$, Francisco Arlysson da Silva Veríssimo ${ }^{1}$, Tobias Júnior do Bomfim Ferreira $^{1}$, Fernanda Nascimento Severo ${ }^{1}$, Alice Maria Correia Pequeno ${ }^{1}$, Ana Patrícia Pereira Morais $^{1}$, Meirecele Calíope Leitinho ${ }^{1}$, Paola Gondim Calvasina ${ }^{1}$.

\section{RESUMO}

Objetivo: relatar uma prática educativa fundamentada na problematização a partir do Arco de Charles Maguerez. Relato da experiência: estudo descritivo, do tipo relato de experiência, envolvendo três mestrandos e dez discentes de uma residência multiprofissional em saúde mental desenvolvido em um Centro de Atenção Psicossocial, realizado de maio a junho de 2018. Inicialmente, foi explicado aos residentes a estratégia problematizadora do Arco de Maguerez e, em seguida, os residentes se dividiram em dois grupos com cinco integrantes em cada para iniciar as cinco etapas do Arco. Os residentes conheceram na aplicação do Arco de Maguerez uma estratégia importante no processo formativo. O emprego desse método ativo de ensino promoveu o estímulo, a autonomia dos residentes e a responsabilização pela construção do conhecimento coletivo. Discussão: a atividade foi avaliada coletivamente pelos participantes, com ênfase na importância de se utilizar metodologias ativas para problematizar uma realidade vivenciada pelos estudantes e construir possibilidades para a transformação da realidade. Considerações finais: $O$ método problematizador do Arco de Maguerez possibilita que seja criada uma rede entre os conhecimentos adquiridos, sendo assim, favorece em uma aprendizagem dirigida e articulada, composta de significados e mediado por novos conceitos, por aproximar o educando ao estudo de uma nova situação.

Palavras-chave: Aprendizagem Baseada em Problemas, Internato e Residência, Metodologia, Saúde Mental.

\begin{abstract}
Objective: To report an educational practice based on problematization from the Charles Maguerez Arch. Experience report: a descriptive, experience report study involving three master's students and ten students from a multiprofessional residency in mental health developed in a Psychosocial Care Center, conducted from May to June 2018. Initially, the residents were explained the strategy Maguerez Arch and then the residents split into two groups with five members each to begin the five stages of the Arch. Residents knew in the application of the Maguerez Arch an important strategy in the formative process. The use of this active method of teaching promoted the stimulation, the autonomy of residents and the responsibility for the construction of collective knowledge. Discussion: the activity was collectively evaluated by participants, emphasizing the importance of using active methodologies to problematize a reality experienced by students and build possibilities for the transformation of reality. Final considerations: The problematizing method of the Maguerez Arch enables a network to be created between the acquired knowledge, thus favoring a directed and articulated learning, composed of meanings and mediated by new concepts, by bringing the student closer to the study of a new situation.
\end{abstract}

Key words: Problem-Based Learning, Internship and Residence, Methodology, Mental Health.

${ }^{1}$ Universidade Estadual do Ceará (UECE), Fortaleza, Ceará. *E-mail: enf.juniorsiqueira@gmail.com 


\section{RESUMEN}

Objetivo: relatar uma prática educativa fundamentada na problemtização a partir del Arco de Charles Maguerez. Relato da experiência: estudo descriptivo, do tipo relato de experiência, envolvendo três mestrandos e dez discentes de uma residência multiprofissional em saúde mental desenvolvido em um Centro de Atenção Psicossocial, realizado de maio a junho de 2018. Inicialmente, foi explicado aos residente. problematizadora do Arco de Maguerez e, em continuación, os residentes se divideiram dois grupos com cinco integrantes em cada para iniciar as cinco etapas do Arco. Los residentes conheceram na aplicação do Arco de Maguerez uma estratégica importante proceso no formativo. O emprego desse método ativo de ensino promoveu o estímulo, a autonomia dos residentes and a responsabilização pela construção do conhecimento coletivo. Discusión: una evaluación de la disponibilidad de los participantes, comience a importar la metodología de utilización de las metodologías ativas para problematizar una realidad de las experiencias de los estudiantes y desarrolle las posibilidades para una transformación de la realidad. Consideraciones finales: El método problematizador del Arco de Maguerez posibilita que seja criada uma rede entre los conhecimentos adquiridos, sendo assim, favorece em uma learningizagem dirigida e articulada, composta de significados e mediado por novos conceitos, por aproximar o educando ao estudo de uma nova situação.

Palabras clave: Aprendizaje Basado en Problemas, Internado y Residencia, Metodología, Salud Mental.

\section{INTRODUÇÃO}

No cenário atual, as Residências Multiprofissionais em Saúde (RMS) constituem uma modalidade de pósgraduação classificada como lato sensu, sendo direcionadas às categorias de profissionais de saúde, voltadas para a educação nos serviços de saúde (CHEADE MFM, et al., 2013).

As RMS foram introduzidas e regulamentadas pela lei no 11.129 , de 30 de junho de 2005. É uma modalidade de formação resultante da união de esforços entre os Ministérios da Educação, Cultura e da Saúde com vistas à criação de estratégias que possibilitem a formação de profissionais aptos para atuarem no Sistema Único de Saúde (SUS) (CHEADE MFM, et al., 2013). Além disso, as residências visam uma formação crítico-reflexiva, de acordo com o que é proposto pela Lei Federal 8080/90, no que se refere à qualificação de recursos humanos para o SUS (CARBOGIM FC, et al., 2010).

Os programas foram estabelecidos com duração mínima de dois anos, devendo ter uma carga horária total de 5.760 horas, sendo $80 \%$ da carga de atividades práticas e $20 \%$ de atividades teóricas ou teórico-práticas, com 60 horas semanais, priorizando atividades em Atenção Primária à Saúde e Hospitalares (SILVA JC, et al.,2015).

A Residência Multiprofissional em Saúde Mental constitui um exemplo de RMS, e atualmente o número de vagas nessa modalidade tem sido crescente. Até o ano de 2010 uma quantidade pequena de vagas era ofertada, cerca de seis em um único estado. No entanto, nos últimos anos houve um aumento na oferta de vagas, alcançando 300 vagas em 29 Programas, distribuídas por 21 cidades em dez estados brasileiros (CAMPOS RO, et al., 2019).

Os cursos de residência têm como objetivo formar profissionais de saúde com vistas a superar a segmentação do conhecimento e do cuidado na atenção em saúde. Assim, utiliza como estratégia de ensinoaprendizagem a formação em serviço, mediante acompanhamento e supervisão. Dessa forma é necessário que os estabelecimentos de saúde e as equipes que trabalham no setor conheçam o programa de residência para que seja possível o funcionamento adequado do estabelecimento como unidade escola (CASONA IA, et al., 2015).

O presente artigo relata uma Prática na Comunidade por meio da utilização de uma metodologia ativa para problematizar a realidade vivenciada por residentes no Centro de Atenção Psicossocial (CAPS) em um município no interior do Ceará. Para problematizar uma realidade, alguns métodos podem ser utilizados, dentre eles as metodologias ativas. O Arco de Charles Maguerez, por exemplo, se baseia como método ativo que tem como ponto de partida a realidade, que ao ser observada por diversos ângulos, permite ao estudante extrair e identificar os problemas existentes (ROCHA R, 2008). 
O presente estudo objetiva relatar uma prática educativa desenvolvida em uma residência multidisciplinar em saúde mental, a qual foi fundamentada segundo os preceitos do método problematizador seguindo as cinco etapas do Arco de Charles Maguerez.

\section{RELATO DE EXPERIÊNCIA}

Trata-se de um estudo descritivo, do tipo relato de experiência, envolvendo 13 participantes, sendo conduzida por três alunos do Curso de Mestrado Profissional Ensino na Saúde da Universidade Estadual do Ceará (UECE) e 10 alunos de uma residência multiprofissional em saúde mental desenvolvida no Centro de Atenção Psicossocial (CAPS) no município de Crateús-Ceará, Brasil, realizado de maio a junho de 2018. Os participantes foram escolhidos por aturem em um estabelecimento de saúde mental.

O trabalho foi desenvolvido como atividade pedagógica de aplicação de estratégias educacionais contemplando os residentes e os mestrandos. A prática na comunidade teve como objetivo a reflexão dos residentes sobre um determinado problema encontrado na realidade do CAPS como ambiente de ensinoaprendizagem no programa de residência. Para realizar a atividade os mestrandos utilizaram o método ativo problematizador denominado Arco de Charlez Maguerez.

A metodologia do Arco de Maguerez segue cinco passos de forma sequencial: Observação da realidade; Identificação dos Problemas (Pontos chaves); Teorização; Hipóteses de solução (Planejamento) e por fim, a Execução da ação (ROCHA R, 2008).

As etapas estabelecidas no Arco de Maguerez contribuem para uma reflexão crítica sobre uma realidade a ser discutida, de modo consciente e intencionalmente transformador, possibilitando um método de trabalho ativo (MELO MC, et al., 2016). O Arco é apresentado na Figura 1.

Figura 1 - Arco de Charles Maguerez. Fortaleza - CE, 2019.

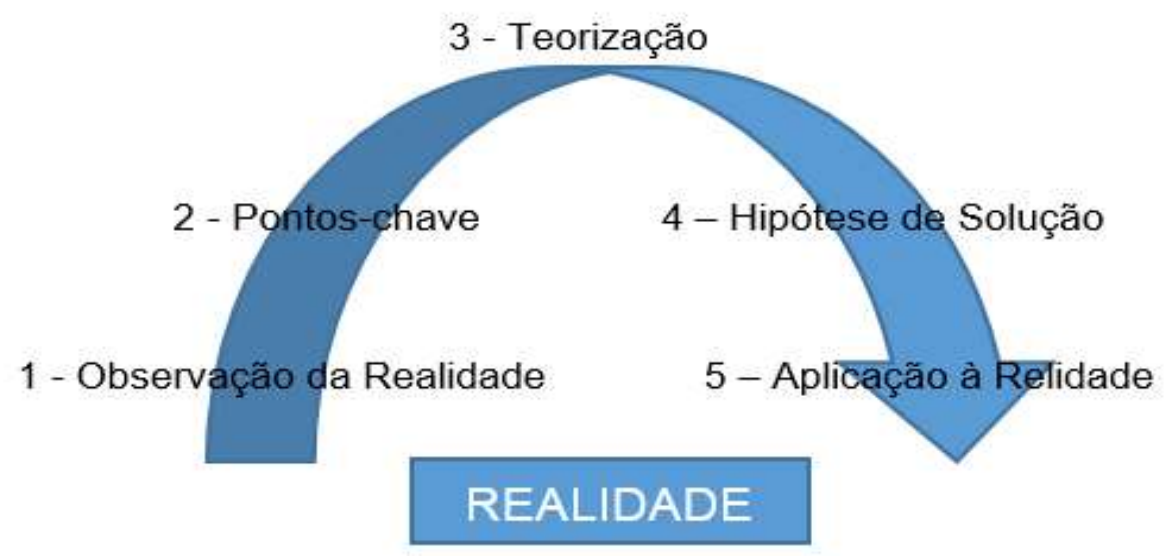

Fonte: Júnior JSA, Veríssimo FAS, Ferreira TJB, et al., adaptado de Berbel CA, Gamboa SAS, 2011.

O presente estudo surgiu por meio de uma atividade avaliativa desenvolvida na disciplina Metodologias Ativas de Ensino-Aprendizagem do Curso Mestrado Profissional Ensino na Saúde da UECE, tendo como objetivo possibilitar que os discentes tivessem maior aproximação e apropriação dos conhecimentos que foram trabalhados durante o módulo do curso. Sendo assim, os mestrandos, atuaram como facilitadores e conduziram os residentes na aplicação do Arco de Maguerez.

Inicialmente fez-se uma explanação sobre Metodologias Ativas e o Arco de Charles Maguerez, ressaltando sua utilização e a importância da participação dos residentes diante das cinco etapas do Arco. Em seguida os residentes foram divididos em dois grupos, com a intenção de explorar as convergências ou diferenças relacionadas à formação em saúde mental entre os estudantes. Em cada grupo foi eleito um relator e um coordenador do processo de discussão e construção do conhecimento. 
A primeira etapa do Arco, observação da realidade, possibilitou uma participação ativa dos residentes para um olhar atento diante da realidade que os mesmos vivenciam na residência. Nesse momento, os discentes passaram a olhar atentamente para a realidade, avaliando e destacando aspectos que necessitavam ser desenvolvidos, trabalhados, revisados ou melhorados. Nessa experiência, a realidade a ser observada foi a residência em saúde mental e o CAPS como Unidade Escola. O problema identificado em ambos os grupos foi a deficiência no processo de ensino-aprendizagem na área da saúde mental no CAPS decorrente da falta de conhecimento dos profissionais que trabalham no estabelecimento sobre o programa de residência em saúde mental.

Na sequência, a segunda etapa possibilitou que os residentes realizassem um levantamento dos pontoschave, ou seja, os fatores que favorecem o problema da realidade. Para a identificação dos pontos, os participantes suscitaram questionamentos que condiziam a discussão, contribuindo para reflexão sobre o tema em questão. Os mestrandos perceberam que os residentes se mostravam integrados e participativos, pois o Arco de Maguerez como metodologia ativa possibilitou discussões e reflexões sobre a residência em saúde mental. Dentre os pontos-chaves destacados pelos participantes estão: falta de organização e preparo da equipe que atua no CAPS, falta de integração com os residentes e o não funcionamento da intersetorialidade.

$\mathrm{Na}$ terceira etapa do Arco, a teorização, os residentes buscaram textos que pudessem estimular reflexões acerca dos pontos-chaves que foram destacados na etapa anterior. Nessa fase ocorreram as operações mentais analíticas que favoreceram o crescimento intelectual dos participantes por meio da leitura de artigos científicos sobre residência em saúde mental. É importante destacar que nessa etapa prevalece o princípio da autonomia e todos os participantes estudaram o assunto (MAIA JÁ, 2014).

As hipóteses de soluções consistem na penúltima etapa do Arco de Maguerez e nela os participantes propuseram alternativas viáveis para solucionar os problemas identificados, de maneira crítica e criativa, a partir do confronto entre teoria e realidade. É uma fase muito importante e que necessita da teorização para que possam ser apresentadas soluções para os pontos-chaves da segunda etapa. Dentre as hipóteses de solução os participantes destacaram: a necessidade de realizar matriciamento das equipes; estabelecer encontros mensais entre a equipe e os residentes; e promover a capacitação da equipe sobre 0 funcionamento do CAPS como unidade escola.

$\mathrm{Na}$ quinta e última etapa do Arco de Maguerez que é a aplicação à realidade, os residentes foram orientados à construir novos conhecimentos para transformar a realidade observada no CAPS, por meio das hipóteses que foram citadas anteriormente. Nessa fase os residentes refletiram sobre o ensino na saúde mental e sobre os principais pontos ligados ao processo de ensino-aprendizagem na residência.

Por fim, os residentes destacaram que o problema evidenciado com a problematização poderia ser solucionado por meio da capacitação da equipe que trabalha no CAPS sobre o funcionamento do estabelecimento como unidade escola na residência em saúde mental. Dessa maneira seriam abordados os principais fatores que necessitam de mudanças para que este setor ofereça com mais qualidade um ambiente apropriado para os residentes diante do processo de ensino-aprendizagem.

Além da capacitação, os residentes também criaram um plano de ação a ser desenvolvido e englobando: rodas de conversa entre os servidores do CAPS, tendo como objetivo discutir os problemas que foram identificados na aplicação do Arco de Maguerez e as possíveis soluções, minicursos e palestras promovidas pelos residentes sobre o CAPS como unidade escola.

A problematização por meio da utilização do Arco de Maguerez foi conduzida tendo como fio condutor o resgate dos conhecimentos prévios dos residentes diante da realidade vivenciada no CAPS. Tal aspecto possibilitou que os participantes desenvolvessem autonomia e responsabilidade frente ao conhecimento que foi construído de forma coletiva. Os residentes que participaram do encontro avaliaram de forma positiva a metodologia que foi utilizada, afirmaram que não conheciam o método do Arco de Maguerez. Referiram que a problematização seguindo as etapas que o compõem possibilitam ao aluno se tornar o ser atuante e agente de mudanças, diante dos mais diversos contextos de atuação. 
A utilização de metodologias ativas de ensino-aprendizagem vem ganhando destaque cada vez mais no contexto atual diante dos benefícios existentes nessa prática. A problematização faz com que os participantes questionem e reflitam sobre muitos aspectos tendo como objetivo apresentar solução diante de um problema encontrado na realidade. A atividade pedagógica da Prática na Comunidade proposta pela disciplina Metodologias Ativas por meio da problematização do Arco de Maguerez possibilitou a construção de um conhecimento articulado, promovendo a autonomia, a reflexão e a criticidade dos residentes diante da realidade vivenciada no CAPS.

Entre as limitações do estudo, pode-se observar que os residentes tiveram dificuldades na utilização do Arco de Maguerez por ser um método aplicado pela primeira vez. No entanto, as dificuldades foram minimizadas pela explicação das etapas e pela compreensão do referencial teórico que fundamenta a problematização.

\section{DISCUSSÃO}

Os residentes seguiram as cinco etapas do Arco, sendo que, durante todo o processo os docentes atuaram como mediadores do processo de ensino-aprendizagem. Na primeira etapa, observação da realidade, os participantes necessitam olhar atentamente para a realidade que vivenciam e destacar o principal problema existente. Nessa fase, o docente pode utilizar algumas estratégias como entrevistas, reportagens, filmes ou até mesmo visitas, as quais possibilitam uma aproximação entre os discentes e a realidade (PRADO ML, et., 2012).

Em seguida, na segunda etapa do Arco, os residentes elencaram os pontos-chave. É nessa etapa onde os discentes iniciam uma reflexão acerca dos fatores determinantes, sendo possível compreender o problema (BERBEL CA; GAMBOA SAS, 2011). Essa etapa é um momento de síntese após a escolha do que será estudado sobre o problema na realidade da residência, os aspectos que precisam ser conhecidos, compreendidos e melhorados (PRADO ML, et., 2012).

Na sequência, durante a terceira etapa do Arco os residentes buscaram textos que estimulassem reflexões acerca da residência em saúde mental. A teorização é um momento em que os residentes passam a compreender o problema e a questionar o porquê dos acontecimentos constatados nas etapas anteriores. Caso esta etapa seja bem desenvolvida leva os residentes a entender o problema, tanto em suas manifestações quanto nos princípios teóricos que os explicam (PRADO ML, et., 2012).

$\mathrm{Na}$ quarta etapa do Arco, os residentes destacaram as Hipóteses de Solução. Nessa fase, por meio de todo o estudo desenvolvida entre os discentes, os mesmos elaboram de maneira crítica e criativa, as possíveis soluções cabíveis de serem aplicadas na realidade (ROCHA R, 2008). Segundo Berbel NAN, (1995), as hipóteses são elaboradas após o estudo como produto da compreensão que se obteve sobre o problema trabalhado.

Por fim, a última etapa desenvolvida pelos residentes foi a aplicação na realidade. Esse momento destinase à prática dos discentes na realidade social presente no CAPS como Unidade Escola. É a fase que possibilita o intervir, o exercitar, o manejar situações que favoreçam na solução do problema. É a etapa em que as soluções do problema estabelecidas pelo grupo são colocadas em prática, a fim de modificar a realidade e o problema identificado no estudo (BERBEL NAN, 1995).

A atividade foi avaliada coletivamente pelos participantes, com ênfase na importância de se utilizar metodologias ativas para problematizar uma realidade vivenciada pelos estudantes e construir possibilidades para a transformação da realidade. O método problematizador do Arco de Maguerez possibilita que seja criada uma rede entre os conhecimentos adquiridos, sendo assim, favorece em uma aprendizagem dirigida e articulada, composta de significados e mediado por novos conceitos, por aproximar o educando ao estudo de uma nova situação (LEAL LB, et al., 2018).

Para que o processo de ensino-aprendizagem se torne efetivo, é necessário que o mesmo seja transformador, sendo que o aluno deve ser o autor responsável pelo próprio processo. Diante disso, é de 
suma importância que os docentes atuem como mediadores na aprendizagem, incentivando a participação e a busca dos educandos por novos conhecimentos, considerando sempre a experiência prévia destes para a produção e apropriação do conhecimento (LEAL LB, et al., 2018).

Assim, a Prática na Comunidade favoreceu o processo de ensino-aprendizagem para mestrandos e residentes, possibilitando o desenvolvimento de competências por meio do treinamento de habilidades com vistas à aprendizagem significativa.

\section{REFERÊNCIAS}

1 BERBEL NAN, GAMBOA SAS. A metodologia da problematização com o Arco de Maguerez: uma perspectiva teórica e epistemológica. Filosofia e Educação, 2011; 3(2):264-89.

2 BERBEL NAN. Metodologia da Problematização: uma alternative metodológica apropriada para o ensino superior. Semina: Cio Soc./Hum, v.16, n.2, p.9-19, 1995.

3 CAMPOS RO, et al. Residência multiprofissional em saúde mental: suporte teórico para percurso formativo. Interface, 2019; 23: e170813.

4 CARBOGIM FC, et al. Residência em enfermagem: a experiência de Juiz de Fora do ponto de vista dos residentes. Revista de Atenção Primária à Saúde, 2010; 13(2): 245-9.

5 CASANOVA IA, et al. Formação para o trabalho em equipe na residência multiprofissional em saúde. Arquivos Brasileiros de Ciências da Saúde, 2015; 40(3):229-33.

6 CHEADE MFM, et al. Residência multiprofissional em saúde: a busca pela integralidade. Revista Cogitare Enfermagem, 2013;18(3):592-5.

7 LEAL LB, et al. Active problematizing method as a strategy for training in health. Revista de Enfermagem UFPE online. 2018;12(4):1139-43.

8 MAIA JA. Metodologias Problematizadoras em currículos de graduação médica. Revista Brasileira de Educação Médica, 2014; 38(4):566-74.

9 MELO MC, et al. Aprendizagem baseada na problematização: utilizando o arco de Maguerez na graduação de enfermagem. Revista Eletrônica Gestão e Saúde, 2016; 7(1):247-59.

10 PRADO ML, et al., Arco de charles maguerez: refletindo estratégias de Metodologia ativa na formação de profissionais de saúde. Escola Anna Nery Revista de Enfermagem, 2012;16(1):172-7.

11 ROCHA R. O método da problematização: prevenção às drogas na escola e o combate a violência. Monografia Universidade Estadual de Londrina; Londrina, 2008; 29p.

12 SILVA JC, et al. Perception of the residents about their performance in the multidisciplinary residency program. Acta Paulista de Enfermagem, 2015; 28(2):132-8. 